OPEN ACCESS

Edited by:

Fengfei $L i$,

Nanjing No. 1 Hospital, China

Reviewed by: Kirtana Nayak,

Manipal Academy of Higher

Education, India

Yan Zhang,

Zhenjiang Fourth People's Hospital,

China

Jufang Wei,

Xiangsi Lake Community Healthcare

Center, China

*Correspondence:

Zilin Sun

sunzilin1963@126.com

Jiangyi Yu

YuJY1961@outlook.com

${ }^{\dagger}$ These authors have contributed equally to this work

Specialty section:

This article was submitted to

Clinical Diabetes,

a section of the journal

Frontiers in Endocrinology

Received: 07 April 2021 Accepted: 04 August 2021 Published: 23 August 2021

Citation:

Chen J, Sun H, Qiu S, Tao H, Yu J and Sun Z (2021) Lipid Accumulation Product Combined

With Urine Glucose Excretion Improves the Efficiency of Diabetes

Screening in Chinese Adults.

Front. Endocrinol. 12:691849. doi: 10.3389/fendo.2021.691849

\section{Lipid Accumulation Product Combined With Urine Glucose Excretion Improves the Efficiency of Diabetes Screening in Chinese Adults}

\author{
Juan Chen ${ }^{1,2+}$, Hong Sun ${ }^{3 \dagger}$, Shanhu Qiu ${ }^{2,4}, \mathrm{Hu} \mathrm{Tao}^{5}$, Jiangyi Yu ${ }^{1 *}$ and Zilin Sun ${ }^{2 *}$
}

\begin{abstract}
${ }^{1}$ Department of Endocrinology, Jiangsu Province Hospital of Chinese Medicine, Affiliated Hospital of Nanjing University of Chinese Medicine, Nanjing, China, ${ }^{2}$ Department of Endocrinology, Zhongda Hospital, Institute of Diabetes, Medical School, Southeast University, Nanjing, China, ${ }^{3}$ Department of Endocrinology and Metabolism, The First Affiliated Hospital of Soochow University, Suzhou, China, ${ }^{4}$ Department of Endocrinology, Shenzhen People's Hospital, The Second Clinical Medical College of Jinan University, The First Affiliated Hospital of Southern University of Science and Technology, Shenzhen, China, ${ }^{5}$ School of Mechanical Engineering, and Jiangsu Key Laboratory for Design and Manufacture of MicroNano Biomedical Instruments, Southeast University, Nanjing, China
\end{abstract}

Background: To compare the efficacy of lipid accumulation product (LAP) and urine glucose excretion (UGE) in predicting diabetes and evaluate whether the combination of LAP and UGE would help to improve the efficacy of using LAP alone or UGE alone in identifying diabetes.

Methods: Data from 7485 individuals without prior history of diabetes who participated in a cross-sectional survey in Jiangsu, China, were analyzed. Each participant underwent an oral glucose-tolerance test. Operating characteristic curves $(R O C)$ and logistic regression analyses were used to evaluate the performance of LAP and UGE in identification of newly diagnosed diabetes (NDM) and prediabetes (PDM).

Results: For subjects with NDM, the area under the ROC curve was 0.72 for LAP and 0.85 for UGE, whereas for PDM, these values were 0.62 and 0.61 , respectively. Furthermore, LAP exhibited a comparable sensitivity with UGE in detecting NDM (76.4\% vs 76.2\%, $\mathrm{p}=0.31$ ). In predicting PDM, LAP showed a higher sensitivity than UGE (66.4\% vs $42.8 \%, p<0.05)$. The combination of LAP and UGE demonstrated a significantly higher sensitivity than that of LAP alone and UGE alone for identification of NDM (93.6\%) and PDM (80.1\%). Moreover, individuals with both high LAP and high UGE had significantly increased risk of NDM and PDM than those with both low LAP and low UGE.

Conclusions: The combination of LAP and UGE substantially improved the efficacy of using LAP and using UGE alone in detecting diabetes, and may be a novel approach for mass screening in the general population.

Keywords: lipid accumulation product, urine glucose excretion, newly diagnosed diabetes, prediabetes, diabetes screening 


\section{INTRODUCTION}

There are evidences that a variety of physiological aberrations caused by obesity mainly depend on the distribution of body fat, instead of overweight or obesity per se $(1,2)$. Of note, it has been reported that visceral fat, but not subcutaneous fat, is strongly associated with metabolic risk factors (3). Computed tomography (CT) or magnetic resonance imaging is considered as the gold standard for assessing lipid accumulation and distribution patterns. Yet they cannot be widely applied in clinical practice since they are costly and time-consuming. In addition, CT examination is radioactive. Lipid accumulation product (LAP), based on a combination of waist circumference (WC) and triglyceride (TG), has been recognized as a novel marker of visceral adipose accumulation (4). The efficacy of LAP for evaluating visceral fat has been demonstrated (5). In addition to the simple calculation, LAP does not require high expenditure of time and cost, thus making it suitable for a large sample population. According to prior research, accumulation of visceral fat measured by CT over 5 years is independently associated with greater risk of incident diabetes (6). Furthermore, several studies have suggested that LAP may serve as a useful predictor of insulin resistance $(4,7)$. Collectively, LAP may be a useful marker to predict diabetes. However, LAP was derived from studies of the US civilian, noninstitutionalized population that included an oversample of non-Hispanic blacks and Mexican Americans. The studies focused on LAP in Chinese population with large sample size are relatively scare.

Due to increased glucose reabsorption, which has been confirmed in individuals with diabetes, the role of kidney in glucose homeostasis has become a research focus in recent years (8). Currently, promoting urine glucose excretion (UGE) through inhibition of renal glucose reabsorption has been demonstrated to be an effective strategy for the treatment of diabetes (9). In addition, UGE reliably reflects the prevailing plasma glucose levels $(10,11)$. In our previous work, we demonstrated that UGE may be a practical and reliable approach for mass screening for diabetes, especially in developing countries (12). Moreover, urine glucose has also been confirmed as the strongest predictor of gestational diabetes due to its noninvasiveness and availability to pregnant women (13). Recently, UGE has gained much more attention because of its significance in clinical practice, such as diabetes screening and glycemic control $(12,14)$. However, its utility for predicting pre-diabetes (PDM), a high-risk state for diabetes, is limited. We found that the sensitivity of UGE for the estimation of $2 \mathrm{~h}-\mathrm{PG} \geq 7.8 \mathrm{mmol} / \mathrm{L}$ was $52.3 \%$, suggesting that nearly half of PDM cases could not be detected by using UGE alone (15). So, we attempted to improve the efficacy of UGE in diabetes screening by introducing new strategies.

Therefore, in the present study, we aimed to confirm the efficacy of LAP in identifying diabetes in Chinese population and compare the efficacy of LAP and UGE in predicting diabetes, and further evaluate whether the combination of LAP and UGE would help to improve the efficacy of using LAP alone or UGE alone in identifying diabetes.

\section{MATERIALS AND METHODS}

\section{Study Design and Participants}

Data were derived from a cross-sectional survey carried out in six cities in Jiangsu Province, China, between November 12, 2015 and June 28, $2016(15,16)$. Individuals aged between 18 and 65 years old, and without prior history of diabetes or taking anti-diabetic medication were invited to take part in this study. This study was conducted in accordance with the 1975 Declaration of Helsinki and was approved by the ethical review committee of Jiangsu Provincial Center for Disease Control and Prevention (JSJK2016 B003 03). Each participant provided written informed consent. A total of 7689 Chinese people participated in the survey. Among them, 204 subjects were further excluded, because they missed the measurement of glycated hemoglobin A1c (HbAlc) or UGE Therefore, 7485 subjects were included in the final analyses (16).

\section{Anthropometric and Laboratory Measurements}

All eligible participants were interviewed using a structured questionnaire to obtain information on demographic characteristics and medical histories. Heart rate (HR), blood pressure (BP), WC, height, and weight were measured with standardized protocols, as previous reported $(17,18)$. Each participant was invited to take an oral glucose tolerance test (OGTT). HbA1c, fasting plasma glucose (FPG), $2 \mathrm{~h}$ plasma glucose (2h-PG), TG, total cholesterol (TC), blood urea nitrogen (BUN), and creatinine were measured. Estimation of glomerular filtration rate (eGFR) was calculated using the CKDEPI equation based on creatinine level (19). The participants were asked to empty their bladders before they were given an OGTT. All the urine samples were collected over a $2 \mathrm{~h}$ period after oral glucose loading. During this period, the participants were not allowed to drink water or undertake strenuous exercise. UGE was calculated as the urine glucose concentration $(\mathrm{mg} / \mathrm{dl}) \times$ urine volume ( $\mathrm{dl})$, as previously described (16). According to previous research, LAP was calculated as $\mathrm{LAP}=(\mathrm{WC}-65) \times \mathrm{TG}$ for men and LAP $=(\mathrm{WC}-58) \times \mathrm{TG}$ for women $(4)$.

\section{Definitions}

Based on the 2012 American Diabetes Association (ADA) criteria, newly diagnosed diabetes (NDM) was defined as the absence of previous diagnosis or treatment for diabetes, and FPG $\geq 7.0 \mathrm{mmol} / \mathrm{L}$ and/or $2 \mathrm{~h}-\mathrm{PG} \geq 11.1 \mathrm{mmol} / \mathrm{L}$ and/or $\mathrm{HbA} 1 \mathrm{c} \geq 6.5 \%$. PDM was defined as $\mathrm{FPG} \geq 5.6 \mathrm{mmol} / \mathrm{L}$ and $\leq$ $6.9 \mathrm{mmol} / \mathrm{L}, 2 \mathrm{~h} P G \geq 7.8 \mathrm{mmol} / \mathrm{L}$ and $<11.0 \mathrm{mmol} / \mathrm{L}$, or $5.7 \% \leq$ HbA1c $\leq 6.4 \%$. LAP exceeding the optimal cutoff point determined in the present study was considered as high LAP (H-LAP), while LAP less than the optimal cutoff point was considered as low LAP (L-LAP). UGE greater than the optimal cutoff point determined in this study was defined as high UGE (H-UGE), otherwise it was considered as low UGE (L-UGE).

\section{Statistical Analysis}

Assuming a sensitivity of $76 \%$, a specificity of $89 \%$, and a disease prevalence of $10 \%$, the allowable error was to 0.1 with $\alpha 0.05$, at 
least 700 subjects were required (12). Continuous variables were presented as means $\pm \mathrm{SD}$, or median (25th to 75th percentiles). Categorical variables were presented as numbers (percentages). The characteristics of the participants in the different groups were compared using one-way analysis of variance (ANOVA) for continuous variables and $\chi 2$ test for categorical variables. Nonparametric Kruskal-Wallis test followed by all pairwise multiple comparisons was performed when the data distribution was skewed. The associations of LAP and UGE with other clinical indicators were examined using Spearman's correlation. Receiver operating characteristic (ROC) curves were generated and the areas under the curves (AUC) were used to evaluate the efficacy of LAP and UGE in detecting NDM and PDM. The sensitivity and specificity were calculated. The optimal cutoff point was determined using the maximum of Youden's index. Logistic regression analyses with adjustment of age, genders, BP, TC, body mass index (BMI), BUN, and creatinine as the confounding factors, were performed to obtain the odds ratios (OR) for NDM and PDM among subjects with H-LAP and H-UGE that was defined according to the cutoff points determined in this study. A $\mathrm{p}$ value $<0.05$ was considered statistically significant. All statistical analyses were conducted using SPSS 22.0 (SPSS Inc, Chicago, IL, USA).

\section{RESULTS}

\section{General Characteristics of the Study Participants}

The general characteristics of the study population, according to glucose tolerance status, are presented in Table 1. Individuals with NDM and PDM were older, had higher BP, FPG, 2h-PG, TC, TG, and showed a greater BMI, compared with normal glucose tolerance (NGT) subjects, while there was no difference in creatinine levels. In addition, individuals with NDM and PDM showed significantly higher LAP in comparison to those with NGT. NDM subjects had highest levels of LAP. Moreover, NDM subjects also exhibited significantly higher UGE than PDM and NGT subjects.

\section{Correlations of LAP and UGE With Glycemic Variables}

LAP was significantly associated with FPG $(r=0.221, \mathrm{p}<0.01)$, 2h-PG ( $\mathrm{r}=0.391, \mathrm{p}<0.01)$, and HbA1c $(\mathrm{r}=0.243, \mathrm{p}<0.01)$ in the overall population. Furthermore, UGE was also positively correlated with FPG $(\mathrm{r}=0.342, \mathrm{p}<0.01), 2 \mathrm{~h}-\mathrm{PG}(\mathrm{r}=0.271, \mathrm{p}<$ $0.01)$, and $\operatorname{HbAlc}(\mathrm{r}=0.253, \mathrm{p}<0.01)$.

\section{Performance of LAP and UGE in Predicting NDM and PDM}

ROC curves represent the diagnostic accuracy of LAP and UGE for the estimation of NDM and PDM. The AUC for LAP was significantly lower than that for UGE for predicting NDM $(\mathrm{P}<$ 0.001), with AUC values of 0.72 (95\%CI: 0.71 - 0.73), 0.85 (95\% CI: 0.85 - 0.86), respectively (Figure 1A). However, LAP showed a comparable sensitivity with UGE in detecting NDM $(\mathrm{P}=0.31)$. LAP displayed a sensitivity of $76.4 \%$ and a specificity of $57.3 \%$ at the optimal cutoff point of 29.6 for the prediction of NDM. Additionally, UGE exhibited a sensitivity of $76.2 \%$ and a specificity of $85.4 \%$ for the detection of NDM at a corresponding optimal cutoff point of 130mg (Table 2).

For identifying PDM, the AUC was 0.62 for LAP and 0.61 for UGE (Figure 1B). The optimal LAP cutoff value in this study population was 21.6 and the corresponding sensitivity and specificity were $66.4 \%$ and $52.0 \%$, respectively (Table 2 ). Moreover, at the optimal UGE cutoff point of $42 \mathrm{mg}$ for PDM,

TABLE 1 | Characteristics of the study participants according to glucose tolerance status.

\begin{tabular}{|c|c|c|c|c|}
\hline & Total $(n=7485)$ & NGT (n = 3243) & PDM (n = 3645) & NDM (n = 597) \\
\hline Age (years) & $43.9 \pm 11.9$ & $39.2 \pm 11.5$ & $47.0 \pm 11.1^{*}$ & $50.0 \pm 9.8^{\star \dagger}$ \\
\hline Male (\%) & 3298 (44.1\%) & $1362(42.0 \%)$ & $1644(45.1 \%)^{\star}$ & $292(48.9 \%)^{*}$ \\
\hline HR (beats/min) & $77.6 \pm 13.3$ & $77.6 \pm 15.3$ & $77.3 \pm 11.5$ & $79.4 \pm 12.2^{\star \dagger}$ \\
\hline \multicolumn{5}{|l|}{ Blood pressure $(\mathrm{mmHg})$} \\
\hline Systolic & $128.3 \pm 19.0$ & $123.3 \pm 17.7$ & $131.0 \pm 18.9^{*}$ & $139.6 \pm 18.4^{\star \dagger}$ \\
\hline Diastolic & $79.2 \pm 14.5$ & $77.0 \pm 17.0$ & $80.3 \pm 11.9^{*}$ & $84.4 \pm 11.3^{\star \dagger}$ \\
\hline \multicolumn{5}{|c|}{ Plasma glucose (mmol/L) } \\
\hline FPG & $5.5 \pm 1.0$ & $5.0 \pm 0.3$ & $5.5 \pm 0.5^{\star}$ & $7.5 \pm 2.1^{* \dagger}$ \\
\hline 2h-PG & $6.6 \pm 2.6$ & $5.5 \pm 1.0$ & $6.6 \pm 1.6^{*}$ & $12.5 \pm 4.4^{\star \dagger}$ \\
\hline HbA1c (\%) & $5.7 \pm 0.6$ & $5.3 \pm 0.2$ & $5.8 \pm 0.3^{*}$ & $7.0 \pm 1.3^{\star \dagger}$ \\
\hline TC (mmol /L) & $4.7 \pm 0.9$ & $4.5 \pm 0.9$ & $4.8 \pm 0.9^{\star}$ & $5.1 \pm 1.0^{* \dagger}$ \\
\hline TG (mmol /L) & $1.2(0.9-1.8)$ & $1.1(0.8-1.6)$ & $1.3(0.9-1.9)^{*}$ & $1.7(1.2-2.5)^{\star \dagger}$ \\
\hline BUN (mmol /L) & $5.0 \pm 1.5$ & $4.8 \pm 1.4$ & $5.2 \pm 1.6^{*}$ & $5.2 \pm 1.5^{\star}$ \\
\hline Creatinine (umol /L) & $74.0(62.0-83.8)$ & $74.2(62.8-84.0)$ & $73.6(62.0-83.2)$ & $73.3(59.8-85.0)$ \\
\hline eGFR $\left(\mathrm{ml} / \mathrm{min} / 1.73 \mathrm{~m}^{2}\right)$ & $96.8 \pm 17.5$ & $99.2 \pm 17.3$ & $95.0 \pm 17.4^{*}$ & $94.4 \pm 17.3^{*}$ \\
\hline BMI & $25.2 \pm 4.0$ & $24.1 \pm 3.8$ & $25.7 \pm 3.9^{*}$ & $27.4 \pm 3.9^{\star \dagger}$ \\
\hline LAP & $26.9(14.3-48.3)$ & 20.8 (11.2 - 38.0) & $30.0(16.9-51.3)^{*}$ & $49.9(30.3-77.9)^{* \dagger}$ \\
\hline UGE (mg) & $28.0(10.0-85.0)$ & $20.0(6.3-48.0)$ & $31.5(11.0-96.0)^{*}$ & $750.0(138.0-1975.0)^{\star \dagger}$ \\
\hline
\end{tabular}

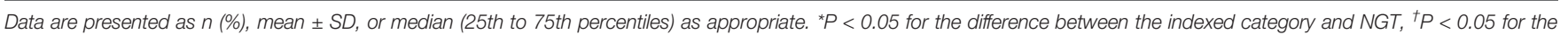

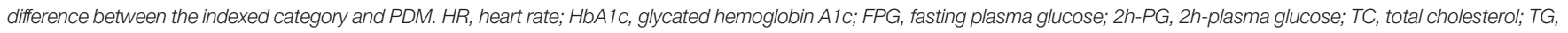

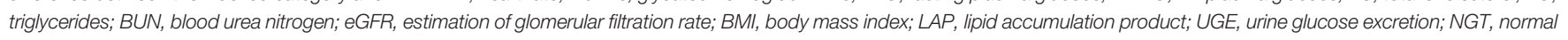
glucose tolerance; PDM, prediabetes; NDM, newly diagnosed diabetes. 
A

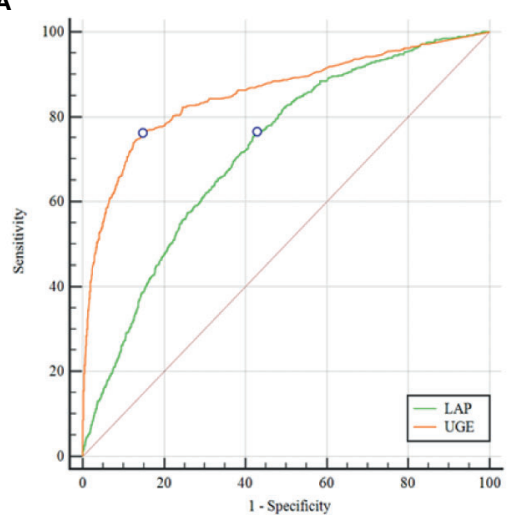

C

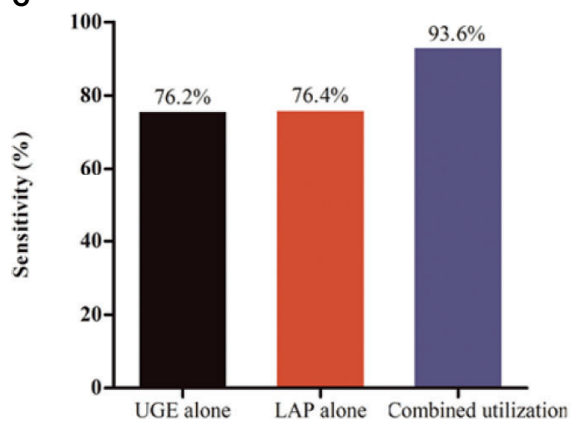

B

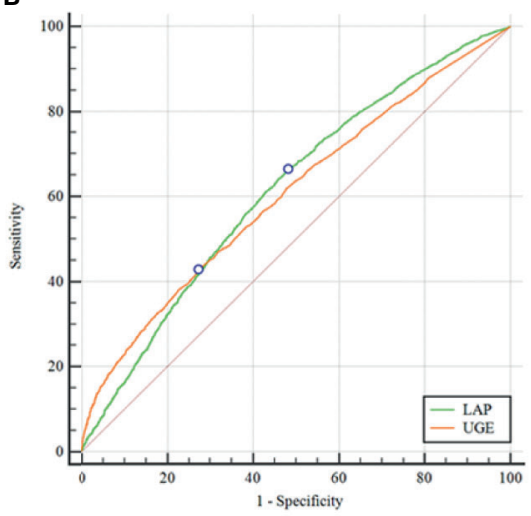

PDM

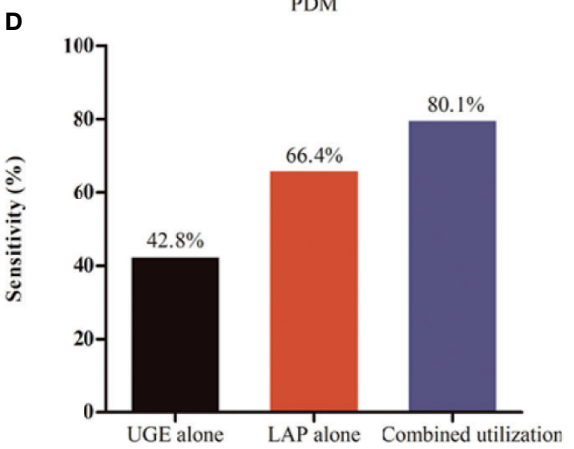

FIGURE 1 | Performance of lipid accumulation product (LAP) and urine glucose excretion (UGE) in predicting for newly diagnosed diabetes (NDM) and prediabetes (PDM). (A) Receiver operating characteristic (ROC) curve for identifying NDM. (B) ROC curve for identifying PDM. (C) Evaluation of LAP combined with UGE for identifying NDM. (D) Evaluation of LAP combined with UGE for identifying PDM.

TABLE 2 | Performance of LAP and UGE for predicting NDM and PDM.

AUC

Optimal
cutoff point

Sensitivity (\%)

Specificity (\%)

\section{For NDM}

LAP $0.72(0.71-0.73) \quad 29.6 \quad 76.4(72.8-79.7) \quad 57.3(56.1-58.5)$

UGE $\quad 0.85(0.85-0.86) \quad 130.0 \quad 76.2(72.6-79.6) \quad 85.4(84.5-86.2)$

For PDM

LAP $0.62(0.60-0.63) \quad 21.6 \quad 66.4(64.8-67.9) \quad 52.0(50.2-53.7)$

UGE $\quad 0.61(0.59-0.62) \quad 42.0 \quad 42.8(41.2-44.5) \quad 72.9(71.3-74.4)$

Data are means (95\% confidence interval). NDM, newly diagnosed diabetes; PDM, prediabetes; LAP, lipid accumulation product; UGE, urine glucose excretion; AUC, the area under the ROC curves.

the sensitivity and specificity were $42.8 \%$ and $72.9 \%$, respectively. LAP $(66.4 \%$, 95\%CI: $64.8 \%$ - 67.9\%) showed a significantly higher sensitivity over UGE (42.8\%, 95\%CI: $41.2 \%-44.5 \%)$ in detecting PDM $(\mathrm{p}<0.05)$.

\section{Evaluation of LAP Combined With UGE in Predicting Diabetes}

As shown in Figures 1C, D, the combined utilization of LAP and UGE showed a significantly higher sensitivity than that of LAP alone and UGE alone for the identification of NDM (both P < 0.001). Further analysis showed that combined utilization of LAP and UGE had an absolute sensitivity advantage of $22.5 \%$ over
LAP alone and $22.8 \%$ over UGE alone. In addition, the sensitivity of using UGE alone to identify PDM was $42.8 \%$, while the combined utilization of LAP and UGE was $80.1 \%$, showing an absolute sensitivity advantage of $87.1 \%$ over UGE alone. Besides, the combined utilization of LAP and UGE had an absolute sensitivity advantage of $20.6 \%$ over LAP alone.

\section{Logistic Regression Analyses of Odds Ratios for NDM and PDM}

Furthermore, binary logistic regression analyses were performed to identify the associations of LAP and UGE with the risk of NDM and PDM. In addition, the joint association of LAP and UGE with the risk of NDM and PDM was assessed by dividing the participants into four groups: L-UGE/L-LAP, L-UGE/H-LAP, $\mathrm{H}-\mathrm{UGE} / \mathrm{L}-\mathrm{LAP}$, and H-UGE/H-LAP. As shown in Figure 2, among the total population, 3405 (45.5\%) participants exhibited H-LAP and 1473 (19.7\%) showed H-UGE. Subjects with H-LAP were more likely to have NDM $(\mathrm{OR}=2.27,95 \% \mathrm{CI}: 1.81-2.83, \mathrm{p}<$ 0.001). Moreover, subjects with H-UGE showed a 17.96-fold increased odds ratio for NDM compared with those with L-UGE. In addition, 3495 (46.7\%) subjects showed both L-LAP and L-UGE, while 888 (11.9\%) displayed both H-LAP and H-UGE. Further analysis showed that individuals with H-UGE/ $\mathrm{H}$-LAP displayed higher risk of NDM $(\mathrm{OR}=36.70,95 \% \mathrm{CI}$ : 25.28 - 53.30) than those with L-UGE/H-LAP (OR $=2.08,95 \% \mathrm{CI}$ : 


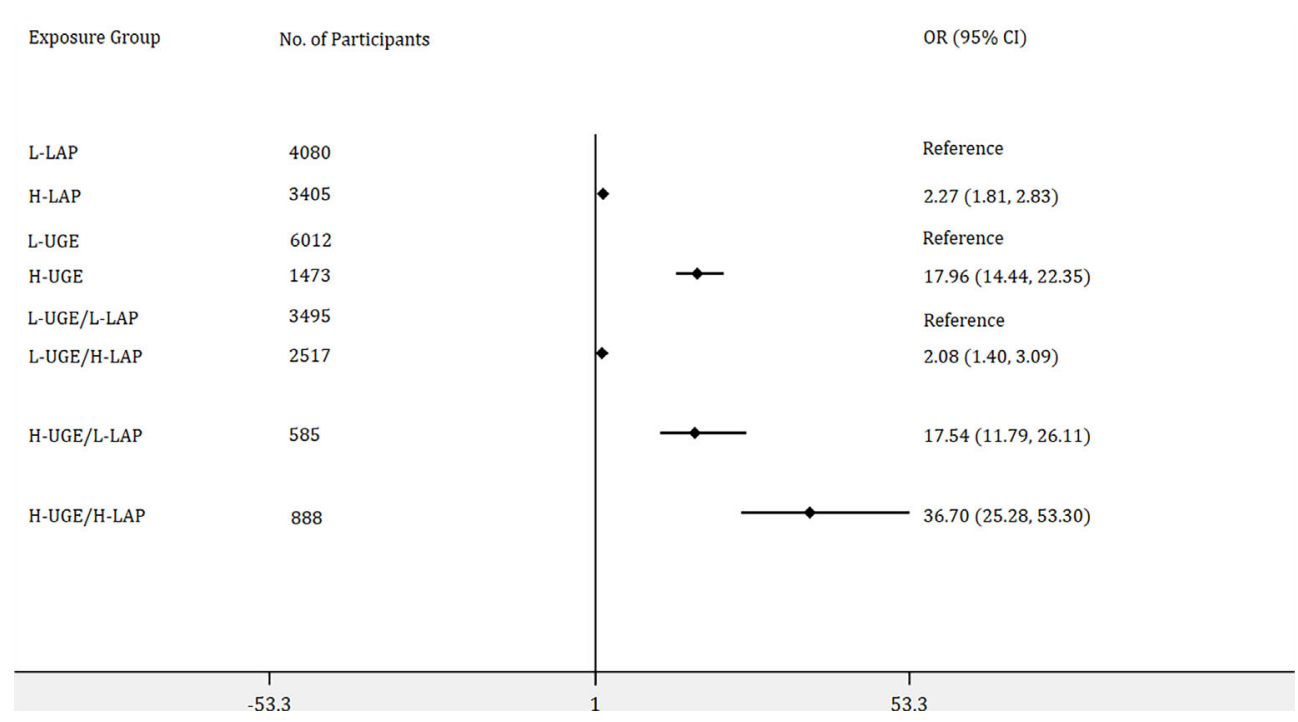

FIGURE 2 | Logistic regression analyses of odds ratios for newly diagnosed diabetes (NDM) with adjustment of age, genders, blood pressure, total cholesterol, body mass index, blood urea nitrogen, and creatinine. The association of lipid accumulation product (LAP) with the risk of NDM was assessed by dividing the participants into two groups: (1) low LAP (L-LAP), (2) high LAP (H-LAP). The L-LAP group was used as a reference in the analysis. The association of urine glucose excretion (UGE) with the risk of NDM was assessed by dividing the participants into low UGE (L-UGE) group and high UGE (H-UGE) group. The L-UGE group was used as a reference in the analysis. In addition, the joint association of UGE and LAP was assessed by dividing the participants into four groups: (1) L-UGE/L-LAP, (2) L-UGE/H-LAP, (3) H-UGE/L-LAP, (4) H-UGE/H-LAP. The L-UGE/L-LAP group was used as a reference in the analysis. The forest plot was displayed in odds ratio and $95 \%$ confidence interval.

$1.40-3.09)$ and H-UGE/L-LAP (OR $=17.54,95 \% \mathrm{CI}$ : 11.79 - 26.11).

As shown in Figure 3, 6888 subjects were identified as nondiabetes, of those, 3970 participants (57.6\%) showed H-LAP and 2491 (36.2\%) exhibited H-UGE. H-LAP and H-UGE were significantly associated with increased odds ratios for PDM. In addition, participants with $\mathrm{H}-\mathrm{UGE} / \mathrm{H}$-LAP displayed the highest risk for PDM $(\mathrm{OR}=2.20,95 \% \mathrm{CI}: 1.86-2.60, \mathrm{p}<0.001)$.

\section{DISCUSSION}

In this study of Chinese participants without previous history of diabetes, we found that UGE showed a better performance in discriminating NDM compared with LAP since the AUC for LAP was significantly lower than that for UGE for detecting NDM $(\mathrm{P}<0.001)$. However, LAP exhibited a comparable sensitivity with UGE in detecting $\mathrm{NDM}(76.4 \%$ vs $76.2 \%, \mathrm{p}=$ 0.31). In addition, in predicting PDM, LAP showed a significantly higher sensitivity than UGE (66.4\% vs $42.8 \%$, p < 0.05). Furthermore, the combined utilization of LAP and UGE improved the sensitivity to $93.6 \%$ for identifying NDM and to $80.1 \%$ for identifying PDM. Since LAP is easy to access and UGE is available, the combined utilization of LAP and UGE might be a practical and sufficient test model for diabetes screening.

The global rise in diabetes has become a public health crisis (20). Notably, accumulating evidences have noted that diabetesassociated complications are sometimes already present in individuals with $\operatorname{NDM}(21,22)$. In addition to health burden, diabetes also leads to a huge economic burden for society (20). Due to its large population, China may bear a higher diabetesrelated burden than any other countries (23). However, according to a prior research, up to $70 \%$ of the people with diabetes are left undiagnosed in China (24). More efficient but inexpensive approaches for predicting diabetes are urgently needed to improve health care for patients with diabetes.

LAP, an index describing the overaccumulation of lipid, is becoming a valuable health screening tool (25). However, LAP was derived from studies of the US civilian, noninstitutionalized population that included an oversample of non-Hispanic blacks and Mexican Americans. The studies that have been designed to focus on the screening performance of this tool in Chinese participants are relatively scare. Our work found that LAP exhibited a sensitivity of $76.4 \%$ for the detection of NDM, indicating that almost $24 \%$ subjects will miss further testing and diagnosis. Besides, a relatively lower sensitivity of $66.4 \%$ for the diagnosis of PDM was observed. However, another study indicated that LAP displayed a sensitivity of $72.3 \%$ for men at the optimal cutoff point of 8.07 , and a sensitivity of $75.1 \%$ for women at the optimal cutoff point of 12.41 , for diagnosis PDM (26). Moreover, our current work indicated that H-LAP was significantly associated with an increased odds ratio of NDM, as well as PDM, which is consistent with recent studies showing that LAP is strongly associated with the risk of type 2 diabetes $(4,27)$. Together, LAP may be a useful clinical marker for the evaluation of hyperglycemia, but the sensitivity can be further improved by introducing new strategies.

It is well known that glycosuria will appear when glycemic excursions exceed the renal threshold for glucose resorption (8). 


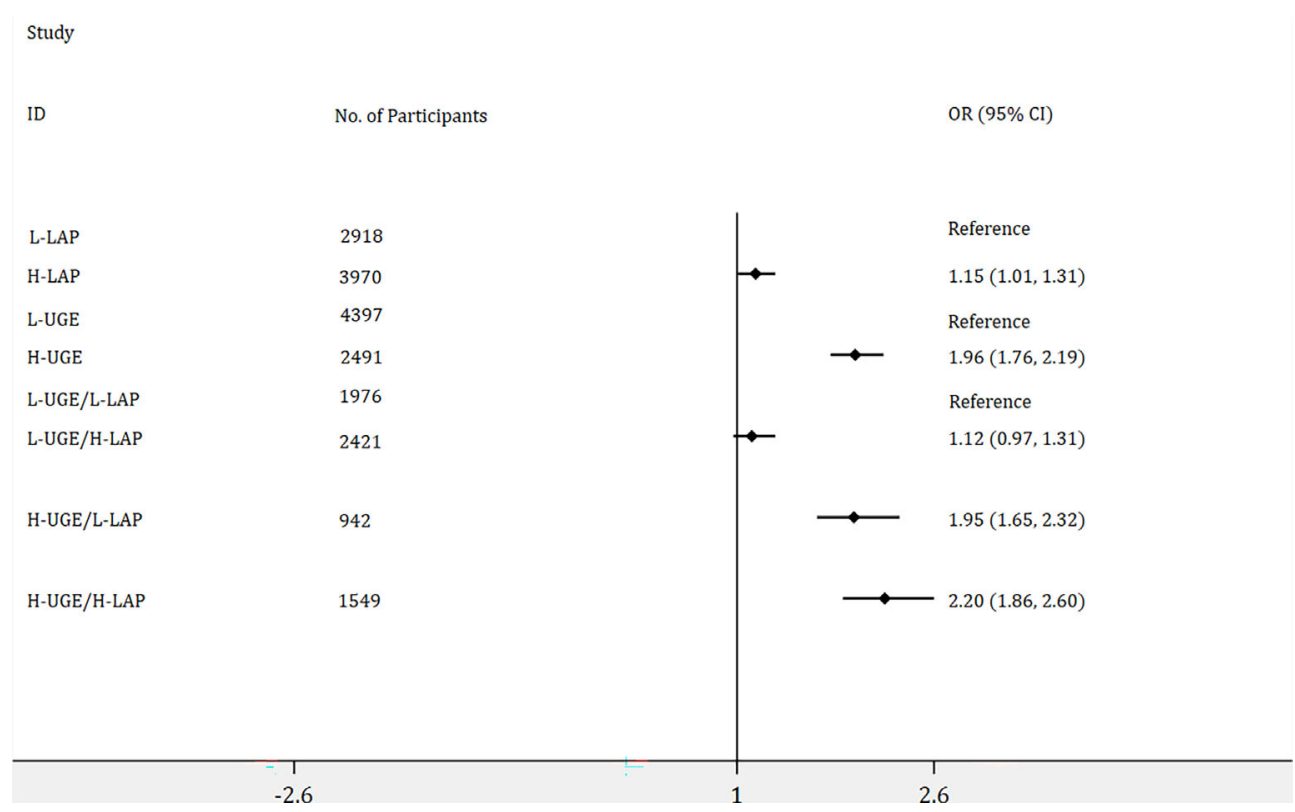

FIGURE 3 | Logistic regression analyses of odds ratios for prediabetes (PDM) with adjustment of age, genders, blood pressure, total cholesterol, body mass index, blood urea nitrogen, and creatinine. The association of lipid accumulation product (LAP) with the risk of PDM was assessed by dividing the participants into two groups: (1) low LAP (L-LAP), (2) high LAP (H-LAP). The L-LAP group was used as a reference in the analysis. The association of urine glucose excretion (UGE) with the risk of PDM was assessed by dividing the participants into low UGE (L-UGE) group and high UGE (H-UGE) group. The L-UGE group was used as a reference in the analysis. The joint association of UGE and LAP was assessed by dividing the participants into four groups: (1) L-UGE/L-LAP, (2) L-UGE/H-LAP, (3) H-UGE/LLAP, (4) H-UGE/H-LAP. The L-UGE/L-LAP group was used as a reference in the analysis. The forest plot was displayed in odds ratio and $95 \%$ confidence interval.

Additionally, based on the findings that UGE reflects the prevailing plasma glucose level and postprandial urine test for glucose is an effective approach for diabetes self-monitoring and selfmanagement $(28,29)$. UGE could be an attractive alternative for identifying hyperglycemia. In the present study, we found positive associations between UGE and glycemic variables, including FPG, 2h-PG, and HbAlc, indicating UGE may be a useful marker for hyperglycemia. Further analyses showed that UGE exhibited a sensitivity of $76.2 \%$ for the detection of NDM, whereas a decreased sensitivity of $42.8 \%$ for the prediction of PDM. Almost $24 \%$ subjects will miss further testing for NDM and almost $58 \%$ subjects will miss further testing for PDM. The efficacy of UGE in PDM screening is limited, in consistent with a previous study (15). However, since UGE is easy to access and inexpensive (12), it should not be completely given up, especially in low-income regions.

We further investigated the sensitivity of LAP in combination with UGE, and a sensitivity of $96.3 \%$ for NDM and $80.1 \%$ for PDM were observed. Our previous wok has demonstrated that determining UGE over a specific period of time is useful for diabetes screening (17). However, the utility of UGE in PDM prediction is limited. Although oral glucose tolerance test (OGTT) is the gold standard for the diagnosis of diabetes, it is impractical to conduct the OGTT for everyone in mass screening due to its complexity (30). Notably, the efficacy of FPG for diabetes screening has been questioned. Since postprandial hyperglycemia is significantly prominent among Chinese patients with diabetes, FPG alone is not sensitive enough $(15,31)$. HbAlc is easy to measure. However, it is also impractical to get all individuals to use
HbAlc because of the relatively high cost, especially in developing countries. In addition, the lack of standardization in $\mathrm{HbAlc}$ assay also limits its use for screening in Chinese population. In the present study, we found that the combination of LAP and UGE substantially improved the efficacy of using LAP alone and using UGE alone for the prediction of diabetes. Since LAP is easy to access and UGE is available, this combination may be a novel approach for mass screening in the general population.

This study was performed in a large Chinese population with no prior history of diabetes. The large sample size provided good power for data analyses. However, the limitations of our study should be considered. First, our population comprised individuals aged between 18 and 65 years old. One might speculate that the impact of LAP and UGE on diabetes diagnosis would have been even stronger in an older population. Thus, generalization of our results to older age groups should be made with caution. Second, our study only involved Chinese Han ethnic subjects, it remains largely unknown whether involving different ethnicities might contribute to differences in sensitivities. Furthermore, in the present study, 66 subjects showed renal function impairment according to the eGFR. The efficacy of UGE for identifying diabetes did not significantly improved after excluding a small number of subjects (data not shown). However, since UGE is mainly mediated by the kidneys, this approach may therefore be inappropriate for those with renal function impairment.

In conclusion, both H-LAP and H-UGE were associated with increased risk for NDM and PDM. Moreover, the combination 
of LAP and UGE substantially improved the efficacy of using LAP alone and using UGE alone in detecting diabetes. Due to its effectiveness, the combined utilization of UGE and LAP may be a novel approach for mass screening in the general population.

\section{DATA AVAILABILITY STATEMENT}

The raw data supporting the conclusions of this article will be made available by the authors, without undue reservation.

\section{ETHICS STATEMENT}

The studies involving human participants were reviewed and approved by The ethical review committee of Jiangsu Provincial Center for Disease Control and Prevention. The patients/ participants provided their written informed consent to participate in this study.

\section{AUTHOR CONTRIBUTIONS}

ZS and JY had full access to all of the data in the study and take responsibility for the integrity of the data and the accuracy of the

\section{REFERENCES}

1. Canoy D, Boekholdt SM, Wareham N, Luben R, Welch A, Bingham, et al. Body Fat Distribution and Risk of Coronary Heart Disease in Men and Women in the European Prospective Investigation Into Cancer and Nutrition in Norfolk Cohort: A Population-Based Prospective Study. Circulation (2007) 116:2933-43. doi: 10.1161/CIRCULATIONAHA.106.673756

2. Després JP, Lemieux I. Abdominal Obesity and Metabolic Syndrome. Nature (2006) 444:881-87. doi: 10.1038/nature05488

3. Ibrahim MM. Subcutaneous and Visceral Adipose Tissue: Structural and Functional Differences. Obes Rev (2010) 11:11-8. doi: 10.1111/j.1467789X.2009.00623.x

4. Brahimaj A, Rivadeneira F, Muka T, Sijbrands E, Franco OH, Dehghan A, et al. Novel Metabolic Indices and Incident Type 2 Diabetes Among Women and Men: The Rotterdam Study. Diabetologia (2019) 62:1581-90. doi: 10.1007/ s00125-019-4921-2

5. Tellechea ML, Aranguren F, Martínez-Larrad MT, Serrano-Ríos M, Taverna MJ, Frechtel GD. Ability of Lipid Accumulation Product to Identify Metabolic Syndrome in Healthy Men From Buenos Aires. Diabetes Care (2009) 32:e85. doi: $10.2337 / \mathrm{dc} 08-2284$

6. Wander PL, Boyko EJ, Leonetti DL, McNeely MJ, Kahn SE, Fujimoto WY. Change in Visceral Adiposity Independently Predicts a Greater Risk of Developing Type 2 Diabetes Over 10 Years in Japanese Americans. Diabetes Care (2013) 36:289-93. doi: 10.2337/dc12-0198

7. Du T, Yuan G, Zhang M, Zhou X, Sun X, Yu X. Clinical Usefulness of Lipid Ratios, Visceral Adiposity Indicators, and the Triglycerides and Glucose Index as Risk Markers of Insulin Resistance. Cardiovasc Diabetol (2014) 13:146. doi: 10.1186/s12933-014-0146-3

8. DeFronzo RA, Hompesch M, Kasichayanula S, Liu X, Hong Y, Pfister M, et al. Characterization of Renal Glucose Reabsorption in Response to Dapagliflozin in Healthy Subjects and Subjects With Type 2 Diabetes. Diabetes Care (2013) 36:3169-76. doi: 10.2337/dc13-0387

9. Ferrannini E. Sodium-Glucose Co-Transporters and Their Inhibition: Clinical Physiology. Cell Metab (2017) 26:27-38. doi: 10.1016/j.cmet. 2017.04.011

10. Miyashita M, Ito N, Ikeda S, Murayama T, Oguma K, Kimura J. Development of Urine Glucose Meter Based on Micro-Planer Amperometric Biosensor and data analysis. JC and HS contributed to the study concept and design, JC, HS, SQ, and TH contributed to the acquisition, analysis and interpretation of the data, JC drafted the manuscript. JC, HS, SQ, TH, ZS, and JY critically revised the manuscript, JC performed the statistical analyses. All authors contributed to the article and approved the submitted version.

\section{FUNDING}

This study was supported by grants from National Key R\&D Program of China (2016YFC1305700), National Key Scientific Instrument and Equipment Development Project of China (No. 51627808), China Postdoctoral Science Foundation (2020M671559), National Natural Science Youth Foundation of China (81700632), Natural Science Youth Foundation of Jiangsu Province (BK20170366).

\section{ACKNOWLEDGMENTS}

We sincerely thank all the staff for their important contributions. We would also like to extend our gratitude to all the enrolled participants.

Its Clinical Application for Self-Monitoring of Urine Glucose. Biosens Bioelectron (2009) 24:1336-40. doi: 10.1016/j.bios.2008.07.072

11. Rave K, Nosek L, Posner J, Heise T, Roggen K, van Hoogdalem EJ. Renal Glucose Excretion as a Function of Blood Glucose Concentration in Subjects With Type 2 Diabetes-Results of a Hyperglycaemic Glucose Clamp Study. Nephrol Dial Transplant (2006) 21:2166-71. doi: 10.1093/ndt/gfl175

12. Chen J, Guo H, Yuan S, Qu C, Mao T, Qiu S, et al. Efficacy of Urinary Glucose for Diabetes Screening: A Reconsideration. Acta Diabetol (2019) 56:45-53. doi: 10.1007/s00592-018-1212-1

13. Lawlor DA, Fraser A, Lindsay RS, Ness A, Dabelea D, Catalano P, et al. Association of Existing Diabetes, Gestational Diabetes and Glycosuria in Pregnancy With Macrosomia and Offspring Body Mass Index, Waist and Fat Mass in Later Childhood: Findings From a Prospective Pregnancy Cohort. Diabetologia (2010) 53:89-97. doi: 10.1007/s00125-009-1560-Z

14. Lu J, Bu RF, Sun ZL, Lu QS, Jin H, Wang Y, et al. Comparable Efficacy of Self-Monitoring of Quantitative Urine Glucose With Self-Monitoring of Blood Glucose on Glycaemic Control in Non-Insulin-Treated Type 2 Diabetes. Diabetes Res Clin Pract (2011) 93(2):179-86. doi: 10.1016/j.diabres .2011.04.012

15. Chen J, Guo HJ, Qiu SH, Li W, Wang XH, Cai M, et al. Identification of Newly Diagnosed Diabetes and Prediabetes Using Fasting Plasma Glucose and Urinary Glucose in a Chinese Population: A Multicenter Cross-Sectional Study. Chin Med J (Engl) (2018) 131:1652-7. doi: 10.4103/0366-6999.235884

16. Chen J, Qiu S, Guo H, Li W, Sun Z. Increased Waist-to-Hip Ratio is Associated With Decreased Urine Glucose Excretion in Adults With No History of Diabetes. Endocrine (2019) 64:239-45. doi: 10.1007/s12020-0181802-2

17. Li W, Xie B, Qiu S, Huang X, Chen J, Wang X, et al. Non-Lab and Semi-Lab Algorithms for Screening Undiagnosed Diabetes: A Cross-Sectional Study. EBioMedicine (2018) 35:307-16. doi: 10.1016/j.ebiom.2018.08.009

18. Chen Y, Li W, Qiu S, Vladmir C, Xu X, Wang X, et al. Tea Consumption and Risk of Diabetes in the Chinese Population: A Multi-Centre, CrossSectional Study. Br J Nutr (2020) 123:428-36. doi: 10.1017/S00071145190 0299X

19. Levey AS, Stevens LA, Schmid CH, Zhang YL, Castro AF3rd, Feldman HI, et al. A New Equation to Estimate Glomerular Filtration Rate. Ann Intern Med (2009) 150:604-12. doi: 10.7326/0003-4819-150-9-200905050-00006 
20. Rodriguez-Gutierrez R, Gonzalez-Gonzalez JG, Zuñiga-Hernandez JA, McCoy RG. Benefits and Harms of Intensive Glycemic Control in Patients With Type 2 Diabetes. BMJ (2019) 367:15887. doi: 10.1136/bmj.15887

21. Jing J, Pan Y, Zhao X, Zheng H, Jia Q, Li H, et al. Prognosis of Ischemic Stroke With Newly Diagnosed Diabetes Mellitus According to Hemoglobin A1c Criteria in Chinese Population. Stroke (2016) 47:2038-44. doi: 10.1161/ STROKEAHA.116.013606

22. Zoppini G, Cacciatori V, Raimondo D, Gemma M, Trombetta M, Dauriz M, et al. Prevalence of Cardiovascular Autonomic Neuropathy in a Cohort of Patients With Newly Diagnosed Type 2 Diabetes: The Verona Newly Diagnosed Type 2 Diabetes Study (VNDS). Diabetes Care (2015) 38:148793. doi: $10.2337 / \mathrm{dc} 15-0081$

23. Yang W, Lu J, Weng J, Jia W, Ji L, Xiao J, et al. Prevalence of Diabetes Among Men and Women in China. N Engl J Med (2010) 362:1090-101. doi: 10.1056/ NEJMoa0908292

24. Xu Y, Wang L, He J, Bi Y, Li M, Wang T, et al. Prevalence and Control of Diabetes in Chinese Adults. JAMA (2013) 310:948-59. doi: 10.1001/jama. 2013.168118

25. Nascimento-Ferreira MV, Rendo-Urteaga T, Vilanova-Campelo RC, Carvalho HB, da Paz Oliveira G, Paes Landim MB, et al. The Lipid Accumulation Product Is a Powerful Tool to Predict Metabolic Syndrome in Undiagnosed Brazilian Adults. Clin Nutr (2017) 36:1693-700. doi: 10.1016/ j.clnu.2016.12.020

26. Nusrianto R, Ayundini G, Kristanti M, Astrella C, Amalina N, Muhadi, et al. Visceral Adiposity Index and Lipid Accumulation Product as a Predictor of Type 2 Diabetes Mellitus: The Bogor Cohort Study of Non-Communicable Diseases Risk Factors. Diabetes Res Clin Pract (2019) 155:107798. doi: 10.1016/j.diabres.2019.107798

27. Yan G, Li F, Elia C, Zhao Y, Wang J, Chen Z, et al. Association of Lipid Accumulation Product Trajectories With 5-Year Incidence of Type 2 Diabetes in Chinese Adults: A Cohort Study. Nutr Metab (Lond) (2019) 16:72. doi: 10.1186/s12986-019-0399-7

28. Dallosso HM, Bodicoat DH, Campbell M, Carey ME, Davies MJ, Eborall HC, et al. Self-Monitoring of Blood Glucose Versus Self-Monitoring of Urine Glucose in Adults With Newly Diagnosed Type 2 Diabetes Receiving
Structured Education: A Cluster Randomized Controlled Trial. Diabetes Med (2015) 32:414-22. doi: 10.1111/dme.12598

29. Müller N, Kämmer K, Kloos C, Wolf G, Müller UA. Postprandial SelfMonitoring of Urine Glucose Reflects Glycaemic Control in People With Relatively Well Controlled Type 2 Diabetes Mellitus Not Treated With Insulin: A Retrospective Cohort Study. Diabetes Med (2015) 32:958-62. doi: 10.1111/dme.12718

30. Wang W, Lee ET, Fabsitz R, Welty TK, Howard BV. Using HbA(1c) to Improve Efficacy of the American Diabetes Association Fasting Plasma Glucose Criterion in Screening for New Type 2 Diabetes in American Indians: The Strong Heart Study. Diabetes Care (2002) 25:1365-70. doi: 10.2337/diacare.25.8.1365

31. Jia WP, Pang C, Chen L, Bao YQ, Lu JX, Lu HJ, et al. Epidemiological Characteristics of Diabetes Mellitus and Impaired Glucose Regulation in a Chinese Adult Population: The Shanghai Diabetes Studies, a Cross-Sectional 3-Year Follow-Up Study in Shanghai Urban Communities. Diabetologia (2007) 50:286-92. doi: 10.1007/s00125-006-0503-1

Conflict of Interest: The authors declare that the research was conducted in the absence of any commercial or financial relationships that could be construed as a potential conflict of interest.

Publisher's Note: All claims expressed in this article are solely those of the authors and do not necessarily represent those of their affiliated organizations, or those of the publisher, the editors and the reviewers. Any product that may be evaluated in this article, or claim that may be made by its manufacturer, is not guaranteed or endorsed by the publisher.

Copyright (c) 2021 Chen, Sun, Qiu, Tao, Yu and Sun. This is an open-access article distributed under the terms of the Creative Commons Attribution License (CC BY). The use, distribution or reproduction in other forums is permitted, provided the original author(s) and the copyright owner(s) are credited and that the original publication in this journal is cited, in accordance with accepted academic practice. No use, distribution or reproduction is permitted which does not comply with these terms. 